\title{
Facile Dashboard Creation Using Library of Syndromic Surveillance Visualization Tools
}

\author{
Harold Gil* \\ Marion County Public Health, Indianapolis, IN, USA
}

\section{Objective}

A framework and toolbox for creating point-and-click dashboard applications (at no cost) for monitoring several facets of syndromic surveillance data was created. These tools (and associated documentation) are being made available freely online for other surveillance practitioners to adopt.

\section{Introduction}

Public health surveillance largely relies on the use of surveillance systems to facilitate the identification and investigation of epidemiologic concerns reflected in data. In order to support public health response, these systems must present relevant information, and be user-friendly, dynamic, and easily-implementable. The abundance of R tools freely-available online for data analysis and visualization presents not only opportunities, but also challenges for adoption in that these tools must be integrated so as to allow a structured workflow. Many public health surveillance practitioners do not have the time available to 1) scavenge for tools, 2) align their functions so as to create a relevant set of visuals, and 3) integrate these visuals into a dashboard that allows a streamlined surveillance workflow. An openly-available, structured framework that allows simple integration of analytic capabilities packaged into readilyimplementable modules would simplify the creation of relevant dashboard visuals by surveillance practitioners.

\section{Methods}

$\mathrm{R}$ is a statistical computing application, known for its versatility and ability to create powerful visualizations. Shiny is an $\mathrm{R}$ package that allows the creation of interactive, easy-to-use point-and-click applications. We looked to $\mathrm{R}$ and its Shiny package extension as a candidate solution. However, creating a Shiny application from scratch requires knowing enough of the $\mathrm{R}$ programming language so as to be able to appropriately design and link several chunks of code that interact with one another to generate the desired output. To address this barrier, we sought to create a structured process by which one can easily browse a library of defined code snippets (each of which enables an analytic tool relevant to syndromic data analysis and visualization) and then integrate snippets of interest into a dashboard application in a way that requisite experience with $\mathrm{R}$ is minimized.

\section{Results}

We first collected several analytic tools that support syndromic data analysis and have been developed for R; examples include heatmaps, change-point detection, outlier detection, tables, maps, etc. We then packaged them into snippets of code (one for each analytic tool) in a way that facilitates integration of the analytic tool into a dashboard application. A fake syndromic dataset was created as well for inclusion in a demo dashboard application that is available for sharing.

\section{Conclusions}

The online community of R users makes new tools for data analysis and visualization available every day. The abundance of options can be overwhelming and the process of integrating pieces of code can be time-consuming. This places a constraint on adoption of these tools by epidemiologists working at all levels of government. The present project alleviates this problem considerably by reducing the tool searching process through the introduction of a library of relevant tools for syndromic data analysis and visualization that can be easily integrated into a dashboard application that allows for streamlined syndromic surveillance activities.

Our next step is to partner with interested jurisdictions to help them adopt this framework and associated tools. Given sufficient interest, we would set up a process for others to add their own modules to this library, perhaps through the online platform for collaborative code development and sharing, GitHub.

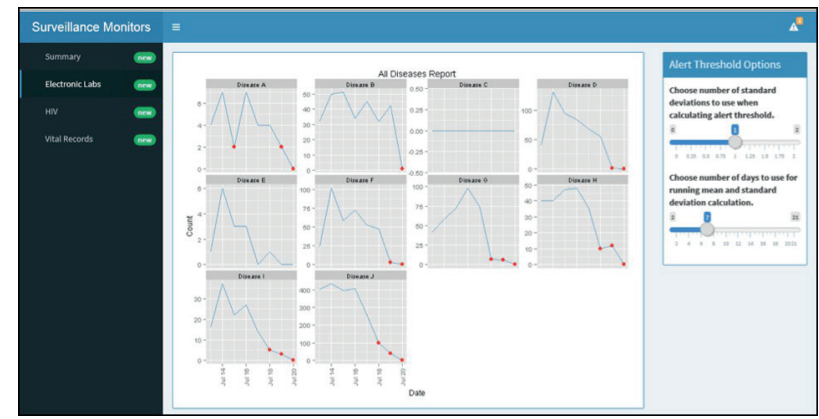

Keywords

Dashboard; Visualization; R

*Harold Gil

E-mail: hgil@marionhealth.org 